University of Nebraska - Lincoln

DigitalCommons@University of Nebraska - Lincoln

\title{
Prediction of the Thermomechanical Behavior of Particle- Reinforced Metal Matrix Composites
}

\author{
Yi Hua \\ University of Nebraska-Lincoln, yhua3@unl.edu \\ Linxia Gu \\ University of Nebraska-Lincoln, gul@fit.edu
}

Follow this and additional works at: https://digitalcommons.unl.edu/mechengfacpub

Part of the Metallurgy Commons, and the Structural Materials Commons

Hua, Yi and Gu, Linxia, "Prediction of the Thermomechanical Behavior of Particle-Reinforced Metal Matrix Composites" (2013). Mechanical \& Materials Engineering Faculty Publications. 84.

https://digitalcommons.unl.edu/mechengfacpub/84

This Article is brought to you for free and open access by the Mechanical \& Materials Engineering, Department of at DigitalCommons@University of Nebraska - Lincoln. It has been accepted for inclusion in Mechanical \& Materials Engineering Faculty Publications by an authorized administrator of DigitalCommons@University of Nebraska Lincoln. 


\title{
Prediction of the Thermomechanical Behavior of Particle-Reinforced Metal Matrix Composites
}

\author{
Yi Hua ${ }^{1}$ and Linxia $\mathrm{Gu}^{1,2}$ \\ 1. Department of Mechanical and Materials Engineering, University of Nebraska-Lincoln, Lincoln, NE 68588-0656, USA \\ 2. Nebraska Center for Materials and Nanoscience, Lincoln, NE 68588-0656, USA \\ Corresponding author - Linxia Gu, Department of Mechanical and Materials Engineering, University of Nebraska-Lincoln, \\ Lincoln, NE 68588-0656, USA; tel 402 472-7680, fax 402 472-1465, email lgu2@unl.edu
}

\begin{abstract}
The objective of this paper was to predict the thermomechanical behavior of 2080 aluminum alloy reinforced with $\mathrm{SiC}$ particles using the Mori-Tanaka theory combined with the finite element method. The influences of particle volume fraction, stiffness, aspect ratio and orientation were examined in terms of effective Young's modulus, Poisson's ratio and coefficient of thermal expansion (CTE) of the composite. The microstructure induced local stress and strain field was obtained through the numerical models of the representative volume element. Results suggested that particle volume fraction had significant impact on the effective Young's modulus, Poisson's ratio and CTE of the composite. Stiffer particles could improve the effective Young's modulus of the composite, while the overall sensitivity of the effective Poisson's ratio and CTE with respect to the particle stiffness was minimal. Particles with larger aspect ratio generally led to a composite with increased effective Young's modulus, as well as reduced Poisson's ratio and CTE. The overall material properties of the composite were insensitive to the particle aspect ratio beyond 10. The particle orientations significantly impacted the effective material properties of the composite, especially along the longitudinal direction. Random 3D dispersed particles exhibited the effective isotropic behavior, whereas anisotropy has been observed for random $2 \mathrm{D}$ and unidirectional aligned particles. Our results could help create tailorable bulk composite.
\end{abstract}

Keywords: discontinuous reinforcement, microstructures, thermomechanical, finite element analysis, micro-mechanics

\section{Introduction}

Particle-reinforced metal matrix composites (MMCs) have been used in space, automotive, and aircraft industries due to their lightweight, high-strength and high-temperature capacity [1-5]. The thermomechanical behavior of this two-phase material is regulated by its microstructures such as particle properties, size and distribution. Several models have been developed to predict the effective material properties of particle-reinforced MMCs using the homogenization theories such as Voigt approximation, Reuss approximation, Mori-Tanaka (M-T) method [6], and the interpolative double-inclusion (DI) method [7]. The M-T theory has been proven to provide reasonable predictions for the effective material properties of two-phase composites. Ziegler et al. [8] used the M-T method to predict the elastic properties of metal/ceramic composites with lamellar microstructures, validated by the experimental data. Doghri and Friebel [9] estimated the effective elastoplastic properties of $\mathrm{SiC}$ whisker-reinforced aluminum matrix composites with various volume fractions, aspect ratios and orientations. The results from the M-T theory were consistent with the experimental data. Duschlbauer et al. [10] presented an extended M-T scheme for modeling the linear thermoelastic and thermophysical behavior of carbon fiber reinforced copper matrix composites, which in agreement with the finite element simulation results. However, the M-T theory failed to provide good estimates for the localization effect induced by the microstructures.

Finite element method (FEM) is capable of identifying the local response of the material. A common practice to estimate the bulk and local responses of composite material is to use a unit cell reinforced by a single fiber, whisker or particle subjected to periodic and symmetric boundary conditions [11-15]. This FE-based approach demands fine mesh at the reinforcement-matrix interface, which led to the need of high end computer as well as costly computation time. Even so, it failed to capture the effect of complex morphology and spatial distribution of the reinforcement, especially for short fiber or irregular particle reinforced matrix. Segurado et al. [16] developed a unit cell model containing 49 particles to investigate the effect of spatial distribution of particles on the elastic response of MMCs. The particles, modeled as spheres, were incorporated with various level of clustering quantified by a radial distribution function. They observed the significant local stress concentrations depending on the level of clustering. Llorca and Segurado [17] used a unit cell model containing 30 non-overlapping identical spheres to study the tensile deformation of particle-reinforced MMCs. Results showed that the strain 


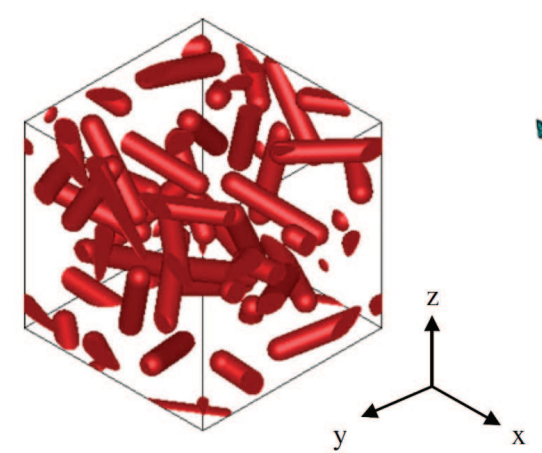

(a)

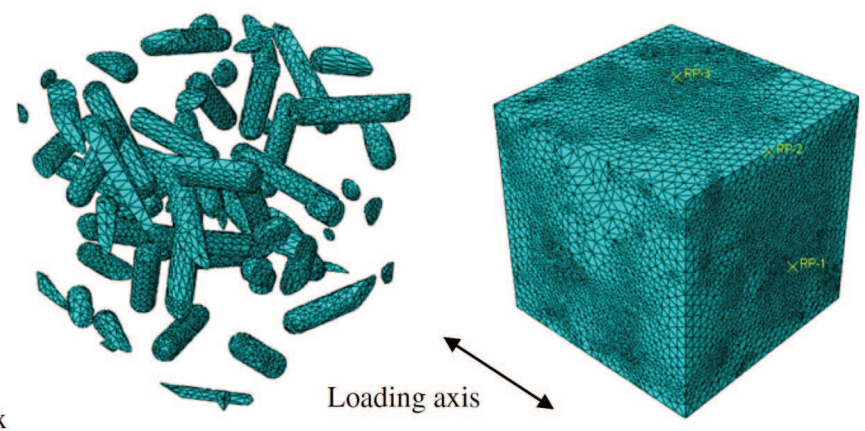

(c)

Figure 1. RVE with random distributed sphero-cylinder shaped particles: (a) geometry, (b) meshed particles only, and (c) meshed RVE.

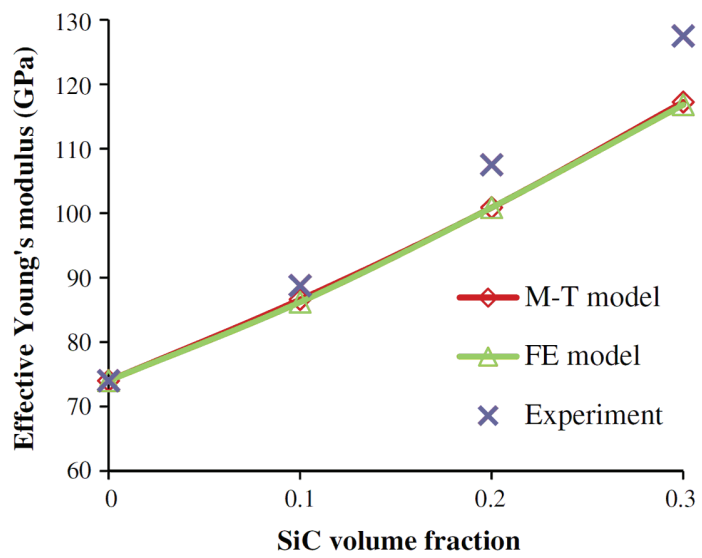

Figure 2. Comparisons among the M-T theory, FE model, and the experimental data [20].

concentration in the matrix was localized in regions between spheres which closely packed along the loading axis. Chawla et al. [18] presented a reconstructed 3D microstructure of SiC particle-reinforced aluminum composites. The localized stress and plasticity was observed due to the sharp and angular nature of irregular particles.

In this work, we combine the computational efficiency of the M- T theory with the local characterization of the FE method to predict the thermomechanical behavior of $2080 \mathrm{alu}-$ minum (Al) alloy reinforced with $\mathrm{SiC}$ particles. A 3D representative volume element (RVE) containing 30 random distributed particles will be developed to estimate the local and global response of MMCs. The influences of particle volume fraction, stiffness, aspect ratio and orientation will be examined in terms of effective Young's modulus, Poisson's ratio and coefficient of thermal expansion (CTE) of the composite. The stress and strain field around microstructures will be used to enhance the understanding of the global mechanical behavior. This work could help engineer to make design decisions on the composite configuration, and guide to engineering a material's microstructure to obtain desirable characteristics.

\section{Finite element modeling}

The microstructure of the 2080 aluminum alloy reinforced with $\mathrm{SiC}$ particles is represented by a three-dimensional RVE ( $1 \mathrm{~mm}$ each side), as shown in Figure 1a. Thirty identical SiC particles are assumed as sphero-cylinder shape and random dispersed. The particle centers are generated using the random sequential adsorption algorithm [19], with identical probability of orientation. The particle diameter depends on the particle volume fraction $V \mathrm{p}$ and aspect ratio $A r$. In the baseline model, the particle volume fraction is set as $10 \%$, and aspect ratio is chosen as 5 . Then the particle diameter is calculated as $0.1 \mathrm{~mm}$. The material properties of $\mathrm{SiC}$ particles are adopted with Young's modulus $E_{\mathrm{p}}=410 \mathrm{GPa}$, Poisson's ratio $v_{\mathrm{p}}=0.19$ and CTE $\alpha_{\mathrm{p}}=4.3 \mathrm{e}^{-6} \mathrm{~K}^{-1}$ [20]. The material properties of $\mathrm{Al}$ alloy matrix are taken from published experimental test as $E_{\mathrm{m}}$ $=74 \mathrm{GPa}, v_{\mathrm{m}}=0.33$ and $\alpha_{\mathrm{m}}=2.5 \mathrm{e}^{-5} \mathrm{~K}^{-1}$ [2]. The two phases are meshed with modified 10-node tetrahedral elements with hourglass control (C3D10M), as shown in Figures 1b and 1c. A mesh convergence study is conducted and the minimum mesh size of $0.05 \mathrm{~mm}$ is chosen. Periodic boundary conditions are imposed to the RVE faces [21]. A uniform $2 \%$ strain is applied to the model along the $x$-direction. A temperature rise of $100{ }^{\circ} \mathrm{C}$ is also applied. The effective material properties of the above described SiC particle-reinforced 2080 aluminum alloy are also predicted using the M-T homogenization theory.

\section{Mori-Tanaka theory}

The considered RVE (domain $\omega$ ) contains a matrix phase, denoted by a subscript 0 , and an inclusion phase, represented by a subscript 1 . The volume fractions $\left(V_{i}\right)$ of these two phases led to $V_{0}+V_{1}=1$.

In isothermal linear elasticity, the mean strain over all inclusions is related by [22]

$$
\langle\varepsilon\rangle_{\omega_{1}}=A^{\varepsilon}:\langle\varepsilon\rangle_{\omega}=B^{\varepsilon}:\langle\varepsilon\rangle_{\omega_{0}}
$$

where a colon designates a tensor product contracted over two indices and the brackets \langle\rangle represent a volume average. $A^{\varepsilon}$ and $B^{\varepsilon}$ are two strain concentration tensors which have the following relationship:

$$
A^{\varepsilon}=B^{\varepsilon}:\left[V_{1} B^{\varepsilon}+\left(1-V_{1}\right) I\right]^{-1}
$$

where I designates the fourth-order symmetric identity tensor. For M-T model, the strain concentration tensor $B^{\varepsilon}$ is given by

$$
B^{\varepsilon}=H^{\varepsilon}\left(I, C_{0^{\prime}} C_{1}\right)=\left\{I+\xi_{\left(I, c_{0}\right)}:\left[\left(C_{0}\right)^{-1}: C_{1}-I\right]\right\}^{-1}
$$

where $\xi_{\left(I, c_{0}\right)}$ is Eshelby's tensor and depends on the geometry of inclusion and matrix stiffness. In thermo-elasticity, the mean strain over all inclusions is related to the macroscopic strain $E$ and a uniform change in temperature $\Delta T$ as

$$
\langle\varepsilon\rangle_{\omega_{1}}=A^{\varepsilon}: E+a^{\varepsilon} \Delta T
$$

with $A^{\varepsilon}$ identical to the linear elastic case and

$$
a^{\varepsilon} \equiv\left(A^{\varepsilon}-I\right):\left(C_{1}-C_{0}\right)^{-1}:\left(\beta_{1}-\beta_{0}\right)
$$




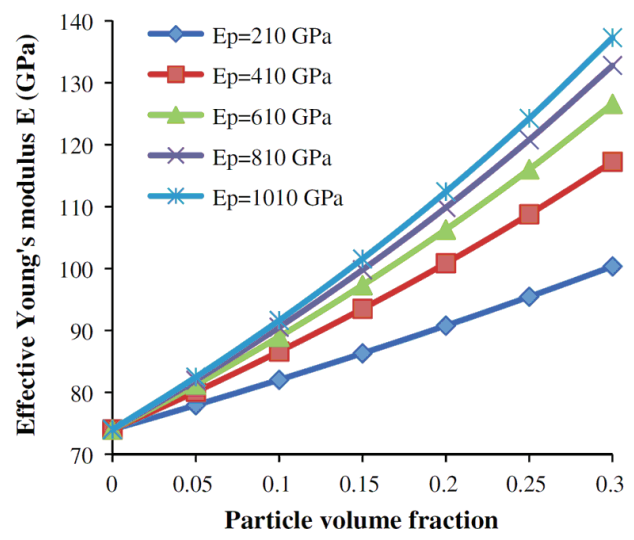

(a)

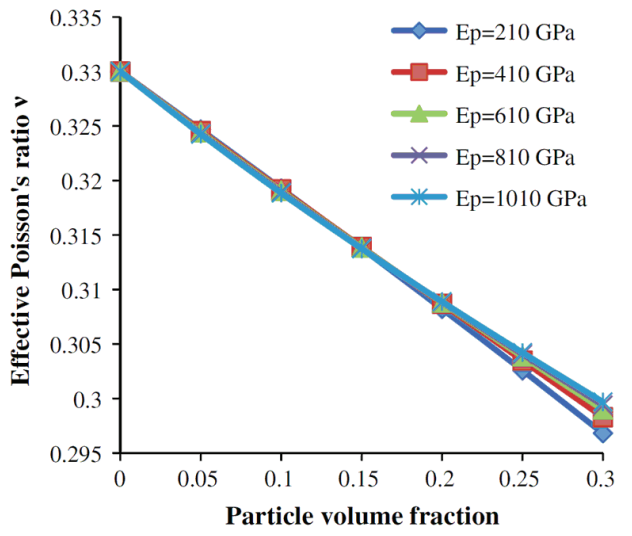

(b)

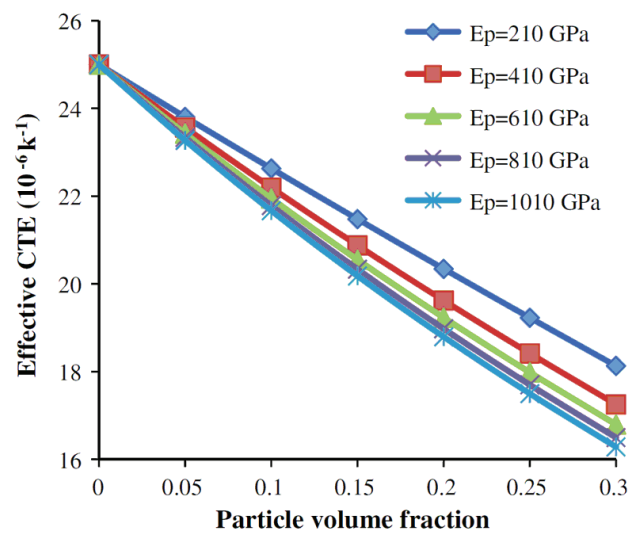

(c)

Figure 3. Variation of the effective material properties with change in particle elastic modulus (particle aspect ratio $A_{\mathrm{r}}=5$ and orientation of random 3D).
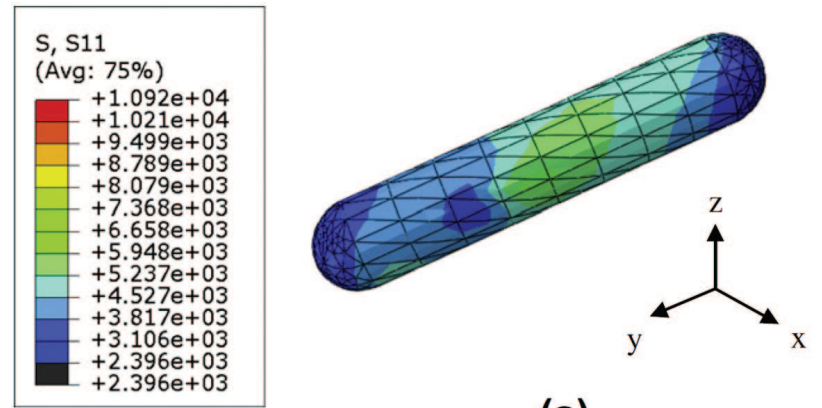

(a)

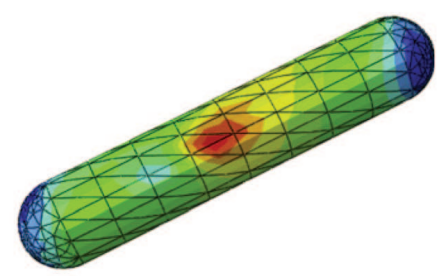

(b)

Figure 4. Stress distribution of $S_{11}$ for particles with elastic modulus: (a) $E_{\mathrm{p}}=410 \mathrm{GPa}$ and (b) $E_{\mathrm{p}}=1010 \mathrm{GPa}$.

$$
E=V_{0}\langle\varepsilon\rangle_{\omega_{0}}+V_{1}\langle\varepsilon\rangle_{\omega_{1}}
$$

where $\beta=-C: \alpha$ and $\alpha$ is the CTE. Per the assumed isotropy, $\alpha_{i j}$ $=\alpha \delta_{i j}$, with $\delta_{i j}$ being Kronecker's symbol.

Then the effective bulk response of the composite can be predicted:

$$
\langle\sigma\rangle=\bar{C}: E+\bar{\beta} \Delta T
$$

where the effective bulk stiffness $\bar{C}$ is obtained as

$$
\bar{C}=\left[V_{1} C_{1}: B^{\varepsilon}+\left(1-V_{1}\right) C_{0}\right]:\left[V_{1} B^{\varepsilon}+\left(1-V_{1}\right) I\right]^{-1}
$$

and the effective CTE as

$$
\bar{\alpha}=-\bar{C}^{-1}: \bar{\beta}
$$

where

$$
\bar{\beta}=V_{0} \beta_{0}+V_{1} \beta_{1}+V_{1}\left(C_{1}-C_{0}\right): a^{\varepsilon}
$$

\section{Results and discussions}

\subsection{Validation}

The published experimental data on the SiC particle-reinforced 2080 aluminum alloy [20] has been used to validate our $\mathrm{M}-\mathrm{T}$ and FE models. The comparative results are shown in Figure 2. It is clear that the predictions of the effective Young's modulus of the composite using both $\mathrm{M}-\mathrm{T}$ and FE models were practically superposed for the particle volume fraction up to $30 \%$. However, the FE analysis was much more time 

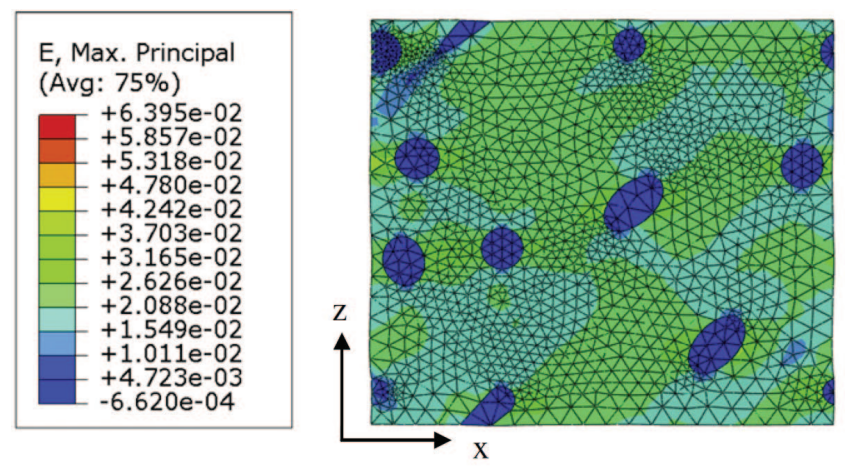

(a)
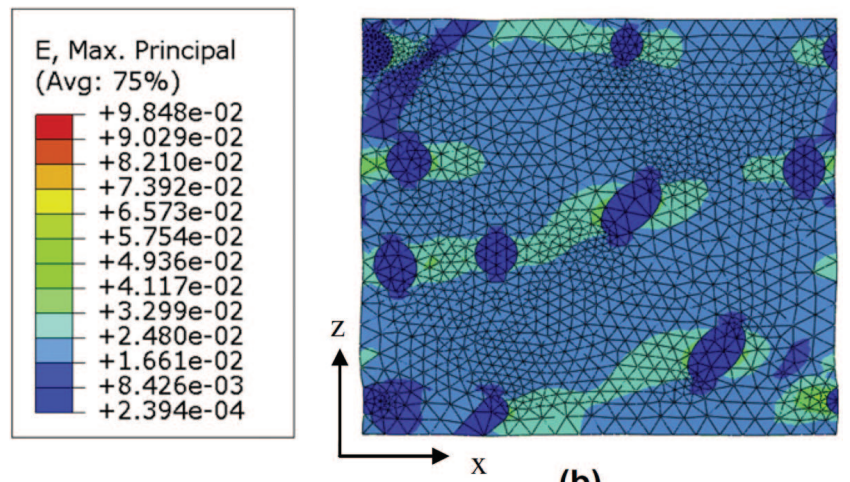

(b)

Figure 5. Maximum principal strain distribution in the RVE with particle modulus: (a) $E_{\mathrm{p}}=410 \mathrm{GPa}$ and (b) $E_{\mathrm{p}}=1010 \mathrm{GPa}$.

consuming, which took over 8750 s for the case of $10 \%$ particle volume fraction running on a Dell T3500 double quadcore Xeon W3550 processor, while it only need less than 1 s using M-T method. The results obtained from both M-T and FE models were lower than the documented experimental results. The discrepancies increased with higher particle volume fraction. It was $8.4 \%$ with the particle volume fraction of $30 \%$. This could attribute to the numerical simplifications such as constant aspect ratio, idealized $3 \mathrm{D}$ random orientation and perfect matrix-particle bonding. A similar underestimate of elastic modulus by simulations has also been observed for the case of unidirectional carbon fiber reinforced silicone matrix subjected to transverse loading [23].

\subsection{Influence of particle stiffness}

For the random dispersed particles with a fixed aspect ratio of 5, the variation of particle materials (elastic modulus $E_{\mathrm{p}}$ ranging from $210 \mathrm{GPa}$ to $1010 \mathrm{GPa}$ ) resulted in the alternations in the effective Young's modulus, Poisson's ratio and CTE of the MMCs (Figure 3). It was observed that the effective Young's modulus of the composite increased with stiffer particle as well as a higher particle volume fraction. The higher sensitivity occurred for particle volume fraction greater than $10 \%$. There was a maximum $36.8 \%$ increase in the effective Young's modulus when particle modulus increased from $210 \mathrm{GPa}$ to $1010 \mathrm{GPa}$ at the $30 \%$ particle volume fraction. The growth rate in effective Young's modulus decreased with the larger particle modulus. For a particle volume fraction of $10 \%$, the growth rate of effective Young's modulus per every $200 \mathrm{GPa}$ was only $1.2 \%$ at the particle modulus of $810 \mathrm{GPa}$, compared to $5.6 \%$ at $210 \mathrm{GPa}$. Minimal sensitivity of the effective Poisson's ratio with respect to the particle modulus was observed, as shown in Figure $3 b$. The total increase of the effective Poisson's ratio was only $1.0 \%$ at the $30 \%$ particle volume fraction when the particle modulus increased from $210 \mathrm{GPa}$ to $1010 \mathrm{GPa}$. The decreasing trend in the effective CTE with the higher particle volume fraction was also observed, as plotted in Figure 3c. The CTE decreased approximately $27.5 \%$ as the particle volume fraction increased from $0 \%$ to $30 \%$. When particle modulus increased from 210 GPa to $1010 \mathrm{GPa}$, there was a maximum $10.2 \%$ reduction in the effective CTE. This could be attributed to the much larger modulus and lower CTE of particles compared to that of metal matrix. The decrease rate of the effective CTE slowed down with the stiffer particle. For the particle volume fraction of $10 \%$, there was only a $0.5 \%$ reduction in CTE during the increase of particle modulus from $810 \mathrm{GPa}$ to $1010 \mathrm{GPa}$, compared to the $1.9 \%$ reduction from $210 \mathrm{GPa}$ to $410 \mathrm{GPa}$.

The influence of particle modulus on the effective Young's modulus, Poisson's ratio, and CTE of the MMCs could be explained by the load sharing capacity of the two-phase material, as presented in Figures 4 and 5, respectively. The microstructures with particle modulus of $410 \mathrm{GPa}$ and $1010 \mathrm{GPa}$ were used to demonstrate the local stress and strain field. The contour plots of the stress component S11 along the loading direction (Figure 4) has shown that the stiffer particles shared more loads when the composite subjected to the same external loading, There was a $74.6 \%$ increase in the peak stress component $S_{11}$ when particle modulus increased from $410 \mathrm{GPa}$ to $1010 \mathrm{GPa}$. The maximum stress occurred in the middle portion of the particle, which was consistent with other local stress observations [24]. It was also noted that the maximum principal strain field in the vicinity of particles was up to three times higher than that far away from the particle-matrix interfaces. This highstrain region due to the interface effect enlarged with the increase of particle modulus. This implies a potential early debonding [25].

\subsection{Influence of particle aspect ratio}

The effective material properties of the composite are also affected by the aspect ratio of the particles $A_{\mathrm{r}^{\prime}}$ defined by the major diameter vs. minor diameter of the particle. Five composites with various particle aspect ratios $\left(A_{\mathrm{r}}=1,5,10,15\right.$ and 20) were considered here under the condition of having fixed particle modulus $E_{\mathrm{p}}=410 \mathrm{GPa}$ and random 3D distribution of particles. The comparative results of M-T method were shown in Figure 6.

Particles with larger aspect ratio proved to be more efficient in composite stiffness enhancement. As particle aspect ratio increased from 1 to 10 with a particle of volume fraction of $30 \%$, there was a maximum $4.5 \%$ increase in the effective Young's modulus, and $1.3 \%$ and $3.1 \%$ of decreases in the effective Poisson's ratio and CTE, respectively. The material properties of the composite were almost unchanged for particle aspect ratio beyond 10. This saturation of reinforcing effect for large particle aspect ratio was also known as the classic "shear-lag" behavior, which described the limiting effect of the small ratio of matrix modulus to particle modulus on the reduction of the load transfer efficiency between phases [26]. In contrast, the effective material properties of MMCs were sensitive to particle volume fraction even with larger aspect ratio. There was a maximum $62.6 \%$ increase, $10.5 \%$ and $32.1 \%$ of decreases in the effective Young's modulus, Poisson's ratio, and CTE, respectively, when particle volume fraction increased from $0 \%$ to $30 \%$ with the particle aspect ratio of 20 .

Probability distributions of stress component $S_{11}$ for particles with aspect ratio $A_{\mathrm{r}}=5$ and $A_{\mathrm{r}}=20$ was obtained from our FE simulations and shown in Figure 7. It was obvious that the most frequently occurring stress for these two cases appeared within the same range, and this peak probability magnitude increased with a larger particle aspect ratio. The maximum probability of $10.7 \%$ occurred at $2125.5 \mathrm{MPa}$ for particles with aspect ratio $A_{\mathrm{r}}=5$ and it was $15.7 \%$ at $2013.5 \mathrm{MPa}$ for particles with aspect ratio $A_{\mathrm{r}}=20$. The tail region of least 


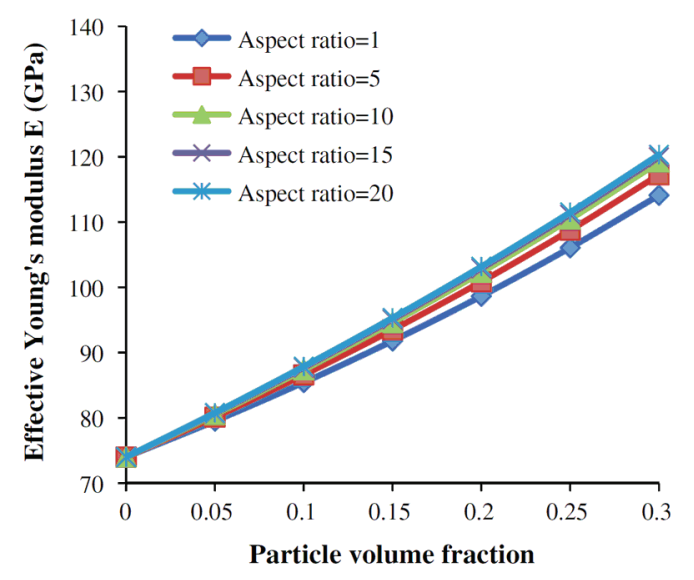

(a)

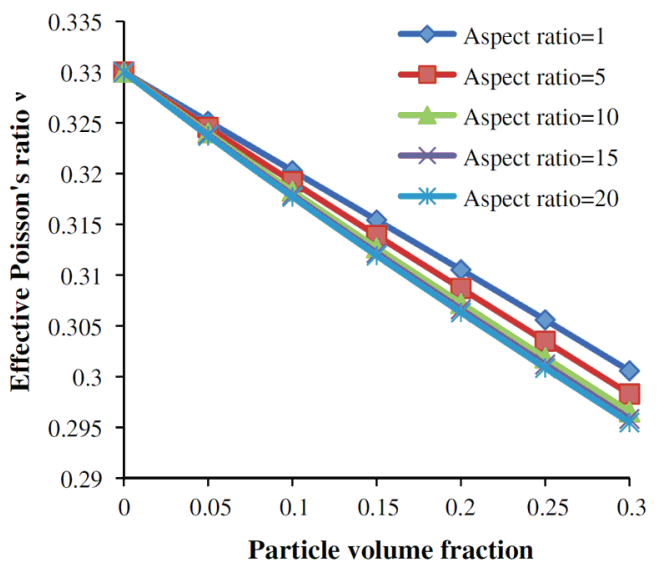

(b)

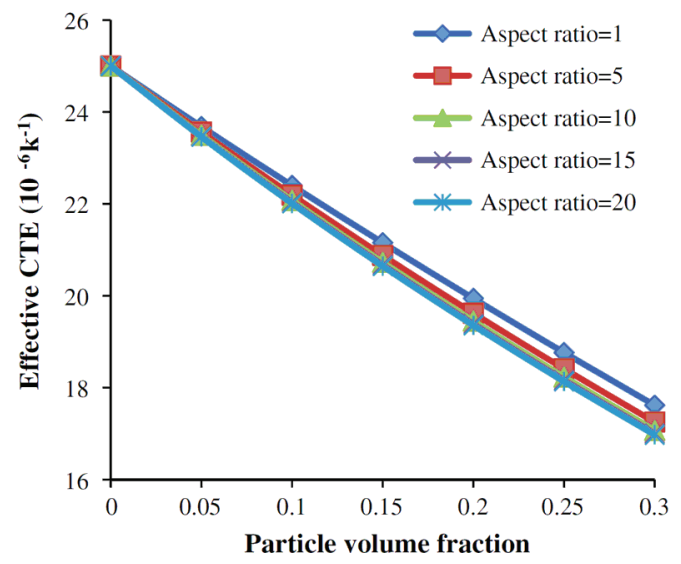

(c)

Figure 6. Variation of the effective material properties with change in particle aspect ratio (particle elastic modulus $E_{\mathrm{p}}=410 \mathrm{GPa}$ and orientation of random 3D).

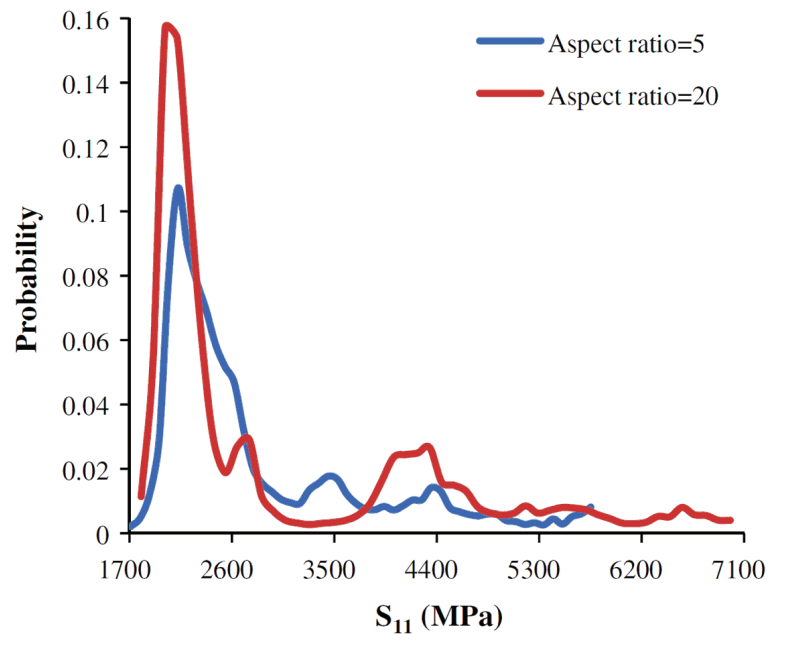

Figure 7. Probability distributions of the stress component $S_{11}$ for particles with aspect ratio $A_{\mathrm{r}}=5$ and $A_{\mathrm{r}}=20$.

frequently occurring stress values for the composite with particle aspect ratio of 20 extended to $6978.6 \mathrm{MPa}$, i.e. the peak stress value of the composite, which was $21.3 \%$ higher than that with aspect ratio of 5 . It demonstrated that the particle aspect ratio significantly altered the peak stress component and the stress distributions.

\subsection{Influence of particle orientation}

The orientation of the dispersed particles is also an important aspect which could influence the effective material properties of the composite. In this section, we investigated three different particle orientations: perfect alignment, also referred to as unidirection, planar orientation state, also called random $2 \mathrm{D}$, and three-dimensional orientation state, also known as random 3D. The results of $\mathrm{M}-\mathrm{T}$ method considering constant particle elastic modulus $E_{\mathrm{p}}=410 \mathrm{GPa}$ and aspect ratio $A_{\mathrm{r}}=5$ were depicted in Figure 8.

Figures $8 \mathrm{a}$ and $8 \mathrm{~b}$ show the influence of particle orientation on the effective Young's modulus of the composites in the longitudinal and transverse directions, respectively. It was noted that the effective modulus in the longitudinal direction $\left(E_{11}\right)$ was strongly dependent on the particle orientation. For the unidirection case, the effective modulus in the longitudinal direction was $19.9 \%$ and $26.4 \%$ higher than the random $2 \mathrm{D}$ and random $3 \mathrm{D}$ cases. However the reinforcement of the longitudinal modulus was at the expense of the transverse modulus. The effective transverse modulus for the unidirection case was reduced by $0.8 \%$ and $4.7 \%$ compared with the random $2 \mathrm{D}$ and random $3 \mathrm{D}$ cases. The dependence of effective modulus on the particle orientation was much weaker in the transverse direction.

The variation of the effective Poisson's ratio of the composite in both the longitudinal and transverse directions with respect to particle orientations was plotted in Figures $8 \mathrm{c}$ and $8 \mathrm{~d}$, respectively. It was interesting to note that, in the case of unidirection case, the effective Poisson's ratio $v_{12}$ increased non- 


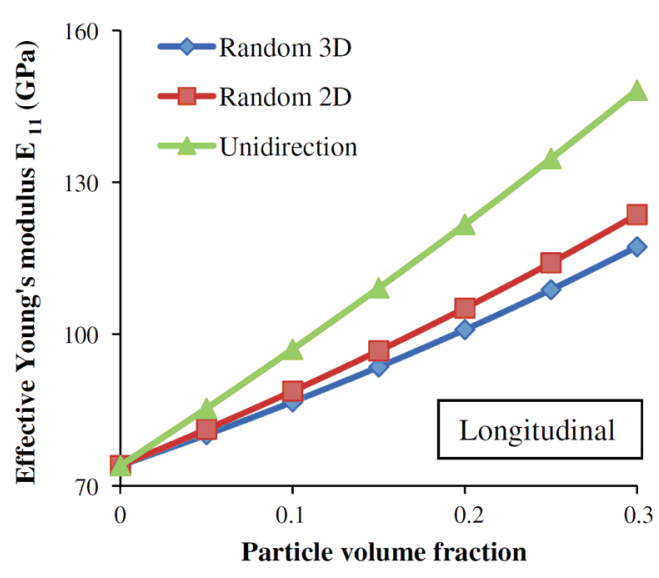

(a)

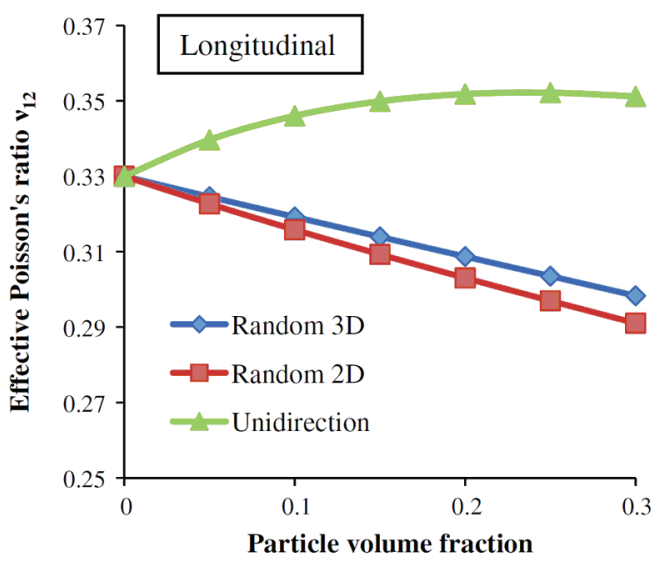

(c)

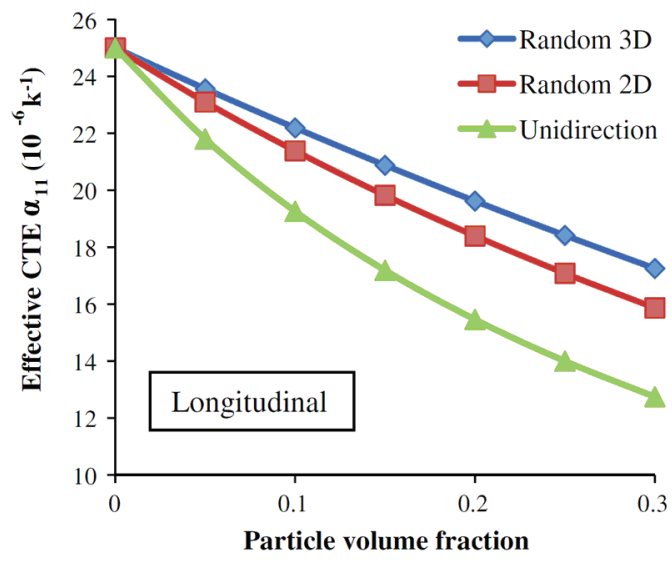

(e)

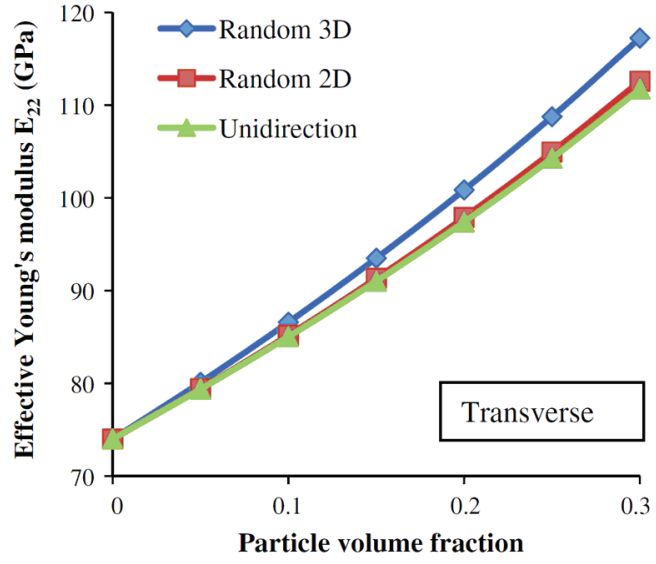

(b)

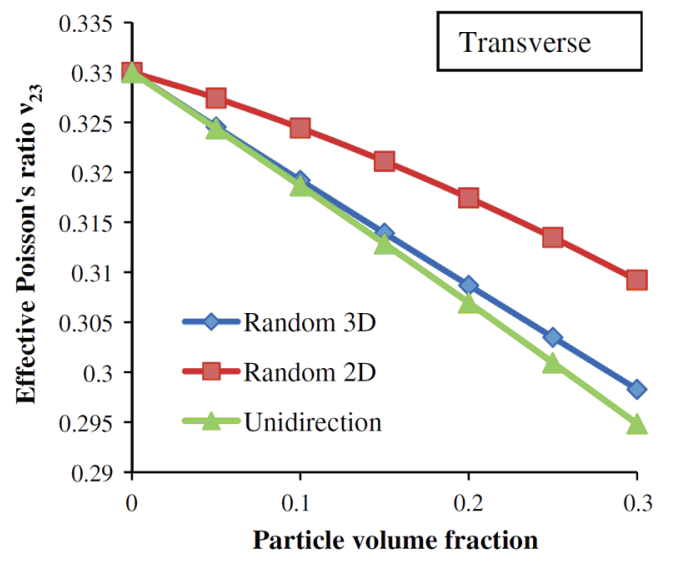

(d)

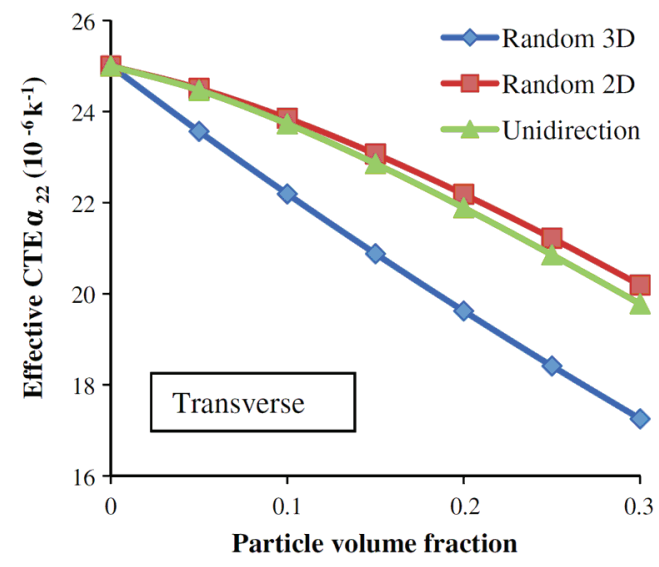

(f)

Figure 8. Variation of the effective material properties with change in particle orientation (particle elastic modulus $E_{\mathrm{p}}=410 \mathrm{GPa}$ and aspect ratio $\left.A_{\mathrm{r}}=5\right)$.

linearly with the increase of particle volume fraction from $0 \%$ to $30 \%$. This nonlinearity was mainly due that the increase in lateral strain was slower than the increase in longitudinal strain. In contrast, this phenomenon did not exist for the 2D or 3D random orientation cases. There was a maximum $11.8 \%$ and $9.6 \%$ decrease of the effective Poisson's ratio $v_{12}$ for $2 \mathrm{D}$ and 3D random orientation cases, respectively. The effective Poisson's ratio $v_{23}$ was less sensitive to particle orientations. Compared to particle orientation with random $2 \mathrm{D}$, there was only a $3.5 \%$ and $4.7 \%$ decrease in $v_{23}$ for random 3D and unidirectional states.

Figures $8 \mathrm{e}$ and $8 \mathrm{f}$ show the predictions of the effective CTE in the longitudinal and transverse directions, respectively. A significant decrease was observed for both $\alpha_{11}$ and $\alpha_{22}$ with the increase of particle volume fraction from $0 \%$ to $30 \%$. The longitudinal CTE a11 was more sensitive to particle orientation than the transverse CTE $\alpha_{22}$. There was a maximum $49.0 \%$ decrease of $\alpha_{11}$ for unidirectional state while the maximum 
decrease of $\alpha_{22}$ was $31.0 \%$ for random 3D state. A definite anisotropy in CTE has been observed for random $2 \mathrm{D}$ and unidirectional orientation states. The difference between the longitudinal CTE a11 and transverse CTE $\alpha_{22}$ in these two cases was $35.6 \%$ and $21.4 \%$, respectively.

\section{Conclusion}

The thermomechanical behavior of 2080 aluminum alloy reinforced with $\mathrm{SiC}$ particles was investigated through the $\mathrm{M}-\mathrm{T}$ method. The RVE models were developed to reveal microstructure induced local stress and strain responses for better interpretation of the behavior of MMCs. The modeling framework was verified by the published experiment [20]. The validated model was then used to further characterize the impact of the particle volume fraction, stiffness, aspect ratio and orientation on the macroscopic properties of MMCs.

It was found that particle volume fraction had significant impact on the thermomechanical behavior of the composite. Stiffer particles could improve the effective Young's modulus, while the overall sensitivity of the effective Poisson's ratio and CTE with respect to the particle stiffness was minimal. Particles with larger aspect ratio generally led to a composite with increased effective Young's modulus, as well as reduced Poisson's ratio and CTE. For particle aspect ratio larger than 10 , the overall material properties were almost unchanged. The particle orientations strongly impacted the effective material properties of MMCs, especially along the longitudinal direction. Random 3D dispersed particles exhibited the effective isotropic behavior, whereas anisotropy has been observed for random 2D and unidirection orientation cases. It should be noted that the enhancement of MMC behaviors due to the particles with increased stiffness or aspect ratio were at the expense of local stress concentrations, which might initiate the failure of materials.

This work provided a fundamental understanding of the impact of microstructure on the bulk behavior of MMCs, which could be used to guide the optimization of the composite configuration to achieve desirable thermomechanical properties.

Acknowledgments - The supports of NASA Nebraska Space Grant are gratefully acknowledged. The authors also thank Mr. Guanfeng Zhang for proofreading this manuscript.

\section{References}

[1] Chen $\mathrm{CH}$, Cheng $\mathrm{CH}$. Effective elastic moduli of misoriented short-fiber composites. Int J Solids Struct 1996;33:2519-39.

[2] Chawla N, Andres C, Jones JW, Allison JE. Effect of SiC volume fraction and particle size on the fatigue resistance of a 2080 Al/SiCp composite. Metall Mater Trans A 1998;29:2843-54.

[3] BanksSills L, Leiderman V, Fang DN. On the effect of particle shape and orientation on elastic properties of metal matrix composites. Compos Part B: Eng 1997;28:465-81.

[4] Liu FR, Chan KC, Tang CY. Numerical modeling of the thermo-mechanical behavior of particle reinforced metal matrix composites in laser forming by using a multi-particle cell model. Compos Sci Technol 2008;68:1943-53.

[5] Chawla N, Shen YL. Mechanical behavior of particle reinforced metal matrix composites. Adv Eng Mater 2001;3:357-70.

[6] Mori T, Tanaka K. Average stress in matrix and average elastic energy of materials with misfitting inclusions. Acta Metall Mater 1973;21:571-4.
[7] Nemat-Nasse S, Hori M. Micromechanics: Overall properties of heterogeneous materials. 2nd ed. Amsterdam: Elsevier Science; 1999.

[8] Ziegler T, Neubrand A, Roy S, Wanner A, Piat R. Elastic constants of metal/ ceramic composites with lamellar microstructures: finite element modelling and ultrasonic experiments. Compos Sci Technol 2009;69:620-6.

[9] Doghri I, Friebel C. Effective elasto-plastic properties of inclusion-reinforced composites. Study of shape, orientation and cyclic response. Mech Mater 2005;37:45-68.

[10] Duschlbauer D, Bohm HJ, Pettermann HE. Computational simulation of composites reinforced by planar random fibers: Homogenization and localization by unit cell and mean field approaches. J Compos Mater 2006;40:2217-34.

[11] Llorca J, Needleman A, Suresh S. An analysis of the effects of matrix void growth on deformation and ductility in metal ceramic composites. Acta Metall Mater 1991;39:2317-35.

[12] Brockenbrough JR, Suresh S, Wienecke HA. Deformation of metal-matrix composites with continuous fibers - Geometrical effects of fiber distribution and shape. Acta Metall Mater 1991;39:735-52.

[13] Shen YL, Finot M, Needleman A, Suresh S. Effective elastic response of 2-phase composites. Acta Metall Mater 1994;42:77-97.

[14] Yuan MN, Yang YQ, Li C, Heng PY, Li LZ. Numerical analysis of the stress-strain distributions in the particle reinforced metal matrix composite SiC/6064Al. Mater Des 2012;38:1-6.

[15] Sun CT, Vaidya RS. Prediction of composite properties, from a representative volume element. Compos Sci Technol 1996;56:171-9.

[16] Segurado J, Gonzalez C, Llorca J. A numerical investigation of the effect of particle clustering on the mechanical properties of composites. Acta Mater 2003;51:2355-69.

[17] LLorca J, Segurado J. Three-dimensional multiparticle cell simulations of deformation and damage in sphere-reinforced composites. Mat Sci Eng A - Struct 2004;365:267-74.

[18] Chawla N, Ganesh VV, Wunsch B. Three-dimensional (3D) microstructure visualization and finite element modeling of the mechanical behavior of $\mathrm{SiC}$ particle reinforced aluminum composites. Scripta Mater 2004;51:161-5.

[19] Widom B. Random sequential addition of hard spheres to a volume. J Chem Phys 1966;44:3888-94.

[20] Chawla N, Sidhu RS, Ganesh VV. Three-dimensional visualization and micro structure-based modeling of deformation in particle-reinforced composites. Acta Mater 2006;54:1541-8.

[21] Reina-Romo E, Sanz-Herrera JA. Multiscale simulation of particle-reinforced elastic plastic adhesives at small strains. Comput Methods Appl Mech 2011;200:2211-22.

[22] Pierard O, Friebel C, Doghri I. Mean-field homogenization of multi-phase thermo-elastic composites: a general framework and its validation. Compos Sci Technol 2004;64:1587-603.

[23] Jimenez FL, Pellegrino S. Constitutive modeling of fiber composites with a soft hyperelastic matrix. Int J Solids Struct 2012;49:635-47.

[24] Sheng N, Boyce MC, Parks DM, Rutledge GC, Abes JI, Cohen RE. Multiscale micromechanical modeling of polymer/ clay nanocomposites and the effective clay particle. Polymer 2004;45:487-506.

[25] Mahmoodi MJ, Aghdam MM, Shakeri M. Micromechanical modeling of interface damage of metal matrix composites subjected to off-axis loading. Mater Des 2010;31:829-36.

[26] Cox HL. The elasticity and strength of paper and other fibrous materials. Brit J Appl Phys 1952;3:72-9. 